\title{
Voclosporin: First Approval
}

\author{
Young-A Heo ${ }^{1}$
}

Published online: 31 March 2021

(c) Springer Nature Switzerland AG 2021

\begin{abstract}
Voclosporin (Lupkynis ${ }^{\mathrm{TM}}$ ) is an oral calcineurin inhibitor immunosuppressant that is being developed by Aurinia Pharmaceuticals. In January 2021, based on positive results from the pivotal phases II and III trials, oral voclosporin received its first approval in the USA for use in combination with a background immunosuppressive therapy regimen for adults with active lupus nephritis. Voclosporin is also being explored for the novel coronavirus disease 2019 (COVID-19) in kidney transplant recipients. This article summarizes the milestones in the development of voclosporin leading to this first approval for lupus nephritis.
\end{abstract}

Digital Features for this AdisInsight Report can be found at https://doi.org/10.6084/m9.figshare.14079959

\section{Voclosporin (Lupkynis ${ }^{\mathrm{Tm}}$ ): Key points}

An oral calcineurin inhibitor immunosuppressant being developed by Aurinia Pharmaceuticals for the treatment of lupus nephritis

Received its first approval on 22 January 2021 in the USA

Approved for use in combination with a background immunosuppressive therapy regimen for adults with active lupus nephritis
This profile has been extracted and modified from the AdisInsight database. AdisInsight tracks drug development worldwide through the entire development process, from discovery, through preclinical and clinical studies to market launch and beyond.

\section{Young-A Heo}

dru@adis.com

1 Springer Nature, Private Bag 65901, Mairangi Bay, Auckland 0754, New Zealand

\section{Introduction}

Lupus nephritis is one of the most serious complications of the systemic lupus erythematosus (SLE), which can cause irreversible and permanent damage to the kidney [1]. The main goal of lupus nephritis treatment is to optimise kidney function, prevent kidney failure and improve patient survival. The 2019 update of the joint European League Against Rheumatism and European Renal Association-European Dialysis and Transplant Association (EULAR/ERA-EDTA) recommends oral mycophenolate mofetil (MMF) or lowdose intravenous cyclophosphamide, both coadministered with glucocorticoids, as initial treatment for active lupus nephritis; however, none are specifically approved for this indication [1]. Moreover, response to treatment is often slow, the risk of relapse is relatively high (18-46\%) and treatment may cause considerate toxicity, making the management of lupus nephritis challenging [2]. Hence, there is an unmet need for effective treatments with improved safety profiles.

Voclosporin (Lupkynis ${ }^{\mathrm{TM}}$ ) is an oral calcineurin inhibitor immunosuppressant developed by Aurinia Pharmaceuticals for the treatment of lupus nephritis. Based on two pivotal phase II and III trials, voclosporin received its first approval in the USA on 22 January 2021 for use in combination with a background immunosuppressive therapy regimen for adults with active lupus nephritis [3]. It is the first oral therapy approved for this indication in the USA [3].

Voclosporin is administered orally in combination with MMF and corticosteroids [4]. The recommended starting dosage of voclosporin is $23.7 \mathrm{mg}$ twice daily with a $12 \mathrm{~h}$ interval (or a minimum of $8 \mathrm{~h}$ interval) between doses as 


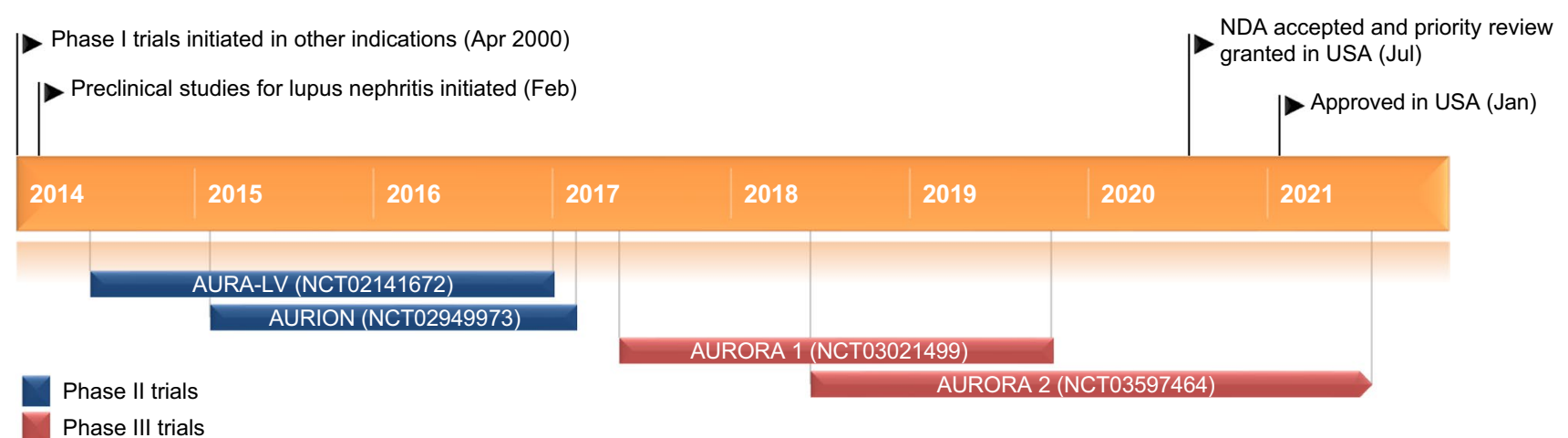

Key milestones in the development of voclosporin for the treatment of lupus nephritis. NDA New Drug Application

possible; the drug is to be swallowed whole on an empty stomach. The dosage of voclosporin is adjusted based on estimated glomerular filtration rate (eGFR); accurate baseline eGFR should be established prior to initiating voclosporin and eGFR should be assessed every 2 weeks for the first month of treatment, and every 4 weeks thereafter. Blood pressure should also be checked prior to treatment and should be monitored every 2 weeks for the first month of treatment and as clinically indicated thereafter. Voclosporin is not recommended for use in patients with a baseline eGFR of $\leq 45 \mathrm{~mL} / \mathrm{min} / 1.73 \mathrm{~m}^{2}$ or those with baseline blood pressure of $>165 / 105 \mathrm{mmHg}$. The US prescribing information contains a boxed warning about the potentially increased risk of developing malignancies and serious infections associated with voclosporin or other immunosuppressants, which can lead to hospitalization or death [4].

Voclosporin is also being explored for the novel coronavirus disease 2019 (COVID-19) in kidney transplant recipients. Clinical evaluation of voclosporin for plaque psoriasis,

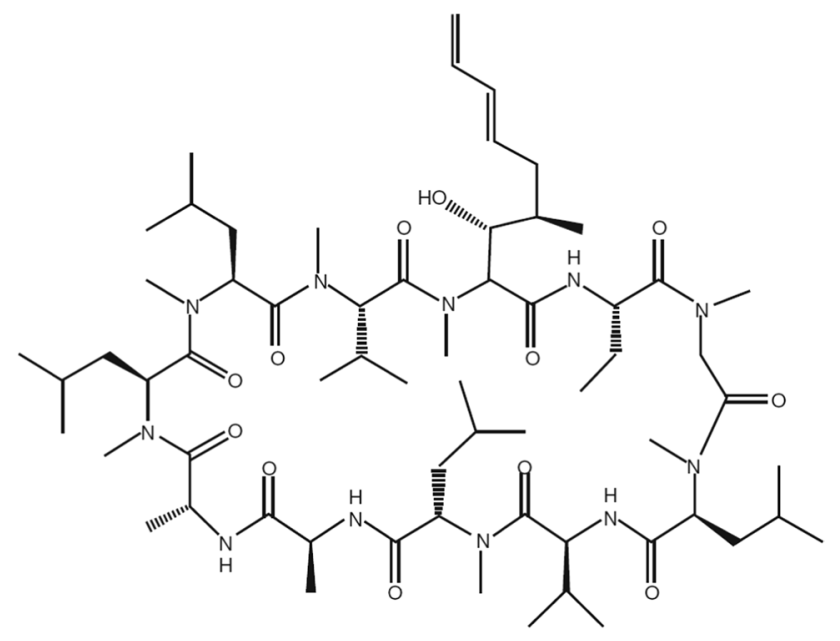

Chemical structure of voclosporin coronary artery restenosis and rheumatoid arthritis has been discontinued and no recent development for prevention of renal transplant rejection has been identified since 2015 .

\subsection{Company Agreements}

Voclosporin was originated and developed by Isotechnika, which subsequently merged with Aurinia Pharmaceuticals [5].

In August 2010, Isotechnika (now Aurinia Pharmaceuticals) entered into an agreement with 3SBio Inc; 3Sbio Inc was granted the exclusive rights to develop and commercialize voclosporin for all transplant and autoimmune disease indications, excluding ophthalmic indications and medical devices, in China, including Hong Kong and Taiwan [6]. In January 2012, Isotechnika (now Aurinia Pharmaceuticals) and Paladin Labs made the last amendment to their plan of arrangement transaction, under which the latter was granted the rights for the marketing of voclosporin in Canada, South Africa and Israel [6].

In February 2013, Isotechnika was merged with Aurinia Pharmaceuticals [5]. Before the merger, ILJIN Life Science held a license for voclosporin, however, as per the tripartite settlement, upon the successful completion of the merger, the combined company reacquired full worldwide rights to voclosporin for all transplant and autoimmune indications, with the exception of Canada, Israel, South Africa, China, Hong Kong and Taiwan. ILJIN Life Science received certain pre-defined future milestone payments and became a founding shareholder in Aurinia Pharmaceuticals [5].

In December 2020, Aurinia Pharmaceuticals and Otsuka Pharmaceuticals entered into a license agreement under which the latter acquired exclusive rights for the development and marketing of voclosporin in Japan and Europe [7]. Under the terms of the agreement, Aurinia Pharmaceuticals will receive an upfront payment and be eligible to receive development and sales-based milestone payments, as well as graduated royalties based on sales levels [7]. 
In December 2020, Aurinia Pharmaceuticals and Lonza expanded an exclusive manufacturing agreement that was first made in December 2016 [8]. Under the expanded agreement, the companies are to build a dedicated capacity within Lonza's existing small molecule active pharmaceutical ingredient facility for the manufacture of voclosporin to expand existing capacity and provide supply security to meet future commercial demand of voclosporin [8].

\subsection{Patent Information}

Isotechnika (now Aurinia Pharmaceuticals) has been granted several patents for composition, formulation and therapeutic use of voclosporin in numerous countries including those of the EU, Australia, Canada, Japan, Morocco, New Zealand, Pakistan, South Africa, South Korea and USA. Upon regulatory approval of voclosporin, patent protection for voclosporin in lupus nephritis is anticipated to be extended to October 2027 in the USA and certain other countries, including those of EU and Japan [9]. In May 2019, Aurinia Pharmaceuticals was granted a US patent for voclosporin entitled "protocol for treatment of lupus nephritis", with a term potentially extending to December 2037 [10].

\section{Scientific Summary}

\subsection{Pharmacodynamics}

Voclosporin is an analogue of cyclosporine with a modification at the amino acid-1 position [11]. The drug has been designed to show improved potency against calcineurin inhibition and better metabolic stability than cyclosporine [11]. Although the exact mechanism of voclosporin is not yet clear, it inhibits calcineurin, thereby blocking lymphocyte proliferation and T-cell mediated immune responses, as well as increasing podocyte integrity in the kidney [4].

Following a single oral administration of voclosporin $0.25-4.5 \mathrm{mg} / \mathrm{kg}$ in healthy subjects, voclosporin inhibited calcineurin activity in a dose-dependent manner up to the dose of $3.0 \mathrm{mg} / \mathrm{kg}$ [12]. There appeared to be little or no lag time between the time taken to reach maximum calcineurin inhibition and the time taken to reach maximum voclosporin concentrations $[4,12]$.

In a randomized, placebo- and moxifloxacin-controlled crossover study, a dose-dependent QT prolongation was observed with single doses of voclosporin $0.5-4.5 \mathrm{mg} /$ $\mathrm{kg}$ (up to 9-fold higher than therapeutic dose), with the time to maximum increase in corrected QT (QTc) interval occurring 4-6 post-dose across dose levels [4]. In a separate, randomized placebo-controlled crossover study in healthy subjects, however, voclosporin $0.3,0.5$ and 1.5 $\mathrm{mg} / \mathrm{kg}(\approx 6$-fold higher than therapeutic dose) twice daily for 7 days were not associated with large mean increases (i.e. > $20 \mathrm{~ms}$ ) in QTc interval [4].

\subsection{Pharmacokinetics}

The pharmacokinetics of voclosporin increases in a greater than dose-proportional manner over the therapeutic range [4]. Steady-state concentrations of voclosporin are achieved after 6 days of twice daily dosing, with an accumulation of $\approx 2$-fold [4].

When administered on an empty stomach, the maximum concentrations $\left(\mathrm{C}_{\max }\right)$ of voclosporin are reached within 1-4 h [4]. In healthy subjects, administration of voclosporin with low- or high-fat meals decreases voclosporin $\mathrm{C}_{\max }$ by $29-53 \%$ and area under the concentration-time curve (AUC) by 15-25\% [12].

Voclosporin has a high protein binding of $97 \%$ and has an apparent volume of distribution of 2154 L [4]. Voclosporin partitions extensively into red blood cells and exhibits concentration- and temperature-dependent distribution between whole blood and plasma [4].

Voclosporin is largely metabolized by CYP3A4, with the parent molecule being the major circulating component [4]. A major metabolite that has $\sim 8$-fold less potency than the parent molecule represented $16.7 \%$ of total exposure in human whole blood. After single oral administration of radiolabelled dose of voclosporin $70 \mathrm{mg}, 92.7 \%$ of radioactivity was recovered from faeces and $2.1 \%$ from urine. The mean apparent clearance of voclosporin at steadystate is $63.6 \mathrm{~L} / \mathrm{h}$ and mean terminal half-life is $\approx 30 \mathrm{~h} \mathrm{[4].}$

There were no clinically relevant effect of age, bodyweight, gender, race, serum albumin, total bilirubin and eGFR on the pharmacokinetics of voclosporin $[4,13]$. Voclosporin $\mathrm{C}_{\max }$ and AUC in patients with mild [creatinine clearance $\left(\mathrm{CL}_{\mathrm{cr}}\right) 60-89 \mathrm{~mL} / \mathrm{min}$ as estimated by Cockcroft-Gault] and moderate $\left(\mathrm{CL}_{\mathrm{cr}} 30-59 \mathrm{~mL} / \mathrm{min}\right)$ renal impairment were similar to those in subjects with normal renal function $\left(\mathrm{CL}_{\mathrm{cr}} \geq 90 \mathrm{~mL} / \mathrm{min}\right)[4,14]$. In patients with severe $\left(\mathrm{CL}_{\mathrm{cr}}<30 \mathrm{~mL} / \mathrm{min}\right)$ renal impairment, voclosporin $\mathrm{C}_{\max }$ and AUC increased by 1.5- and 1.7-fold, respectively. Voclosporin $\mathrm{C}_{\max }$ and AUC increased by $\approx 1.5$ - to 2.0-fold in patients with mild (Child-Pugh A) or moderate (ChildPugh B) hepatic impairment $[4,14]$. A reduced starting dosage of voclosporin $15.8 \mathrm{mg}$ twice daily is recommend in patients with severe renal impairment or mild to moderate hepatic impairment [4]. The effect of end-stage renal disease with or without haemodialysis and severe (Child-Pugh C) hepatic impairment on the pharmacokinetics of voclosporin is unknown; voclosporin is not recommended in patients with severe hepatic impairment [4]. 
Coadministration of voclosporin with strong (e.g. ketoconazole, itraconazole, clarithromycin) or moderate (e.g. fluconazole, diltiazem) CYP3A inhibitors increase voclosporin $\mathrm{C}_{\max }$ and $\mathrm{AUC}$, while coadministration with potent (e.g. rifampin) or moderate (e.g. efavirenze) CYP3A inducers decrease voclosporin $\mathrm{C}_{\max }$ and AUC $[4,15]$. Coadminstration of voclosporin with strong CYP3A4 inhibitors should be avoided since coadministration can significantly increase voclosporin exposure, potentially increasing the risk of acute and/or chronic nephrotoxicity; dosage adjustment is recommended when voclosporin is coadministered with moderate CYP3A4 inhibitors [4]. Voclosporin had no clinically relevant effect on $\mathrm{C}_{\max }$ and $\mathrm{AUC}$ of the sensitive CYP3A4 substrate midazolam but increased $\mathrm{C}_{\max }$ and AUC of P-gp substrate digoxin as it is a weak P-gp inhibitor; thus, if $\mathrm{P}$-gp substrates with narrow therapeutic indices are coadministered with voclosporin, dose reduction of $\mathrm{P}-\mathrm{gb}$ substrates may be required. In vitro, voclosporin is not a substrate of BCRP, OATP1B1 and OATP1B3 but an inhibitor of OATP1B1 and OATP1B3 [4].

Coadministration of voclosporin $23.7 \mathrm{mg}$ twice daily with MMF had no effect on MMF exposure in 25 patients with SLE with or without lupus nephritis[16]. Concomitant use of voclosporin with cyclophosphamide is not recommended as the efficacy and safety of this combination has not be established [4].

\subsection{Therapeutic Trials}

Voclosporin in combination with MMF plus corticosteroids significantly improved renal function in patients with active lupus nephritis in the randomized, double-blind, placebocontrolled phase III AURORA 1 (NCT03021499) trial [17-19]. Patients with active lupus nephritis [i.e. kidney biopsy-proven active class III or IV lupus nephritis with a urine protein to creatinine (UPCR) ratio of $\geq 1.5 \mathrm{mg} / \mathrm{mg}$ or class V lupus nephritis with a UPCR ratio of $\geq 2.0 \mathrm{mg} /$ $\mathrm{mg}$ ] were randomized to receive voclosporin $23.7 \mathrm{mg}$ twice daily $(n=179)$ or placebo $(n=178)$, in addition to standardof-care therapy (i.e. MMF 2 g/day and low dose oral steroids tapered down to $2.5 \mathrm{mg} /$ day), for 52 weeks [17-19]. At week 52, the proportion of patients achieving complete renal response (primary endpoint) was significantly $(p<0.0001)$ higher with add-on voclosporin than with placebo [40.8\% vs $22.5 \%$; odds ratio (OR) $2.65,95 \%$ CI $1.64-4.27]$. Complete renal response was defined as UPCR of $\leq 0.5 \mathrm{mg} / \mathrm{mg}$, confirmed eGFR of $\geq 60 \mathrm{~mL} / \mathrm{min}$ per $1.73 \mathrm{~m}^{2}$ or no $>20 \%$ decrease in eGFR from baseline, presence of sustained, low dose steroids (i.e. no more than $10 \mathrm{mg}$ prednisone equivalent per day for $\geq 3$ consecutive days or for $\geq 7$ days during the 8 weeks prior to endpoint assessment) and no administration of rescue medication. In addition, all pre-specified hierarchical secondary endpoints of renal response at week 24, partial renal response at weeks 24 and 52, time to UPCR $\leq 0.5 \mathrm{mg} / \mathrm{mg}$ and time to $50 \%$ reduction in UPCR from baseline significantly $(p \leq 0.002)$ favoured add-on voclosporin over placebo [17-19]. The treatment benefit of add-on voclosporin over placebo was consistently seen regardless of kidney biopsy class, age, sex, race, region and prior use of MMF $[18,19]$.

In the 48-week, randomized, double-blind, placebo-controlled phase II AURA-LV (NCT02141672) trial in patients with active lupus nephritis, a significantly greater proportion of patients achieved complete renal response at week 24 (primary endpoint; defined as in AURORA 1) with low-dose voclosporin plus standard-of care therapy $(23.7 \mathrm{mg}$ twice daily; $n=89$ ) than with placebo plus standard-of care therapy $(n=88)[32.6 \%$ vs $19.3 \%$; OR $2.03,95 \%$ CI $1.01-4.05$; $p=0.046$ [ [20]. However, statistical significance was not reached with high-dose voclosporin plus standard-of care (39.5 mg twice daily; $n=88$ ) versus placebo plus standardof care $(27.3 \%$ vs $19.3 \% ; 1.59,0.78-3.27 ; p=0.204)$. The

Features and properties of voclosporin

Alternative names

Class

Mechanism of Action

Route of Administration

Pharmacodynamics

Pharmacokinetics

Adverse events (>10\%)

ATC codes

WHO ATC code

EphMRA ATC code

Chemical Name
trans-ISA 247; trans-R 1524; ISA(TX)247; ISA-247; ISAtx 247; ISATx247; LUPKYNIS; Orelvo; R-1524; Voclera Anti-inflammatories, antipsoriatics, antirheumatics, antivirals, ciclosporins, eye disorder therapies, immunotherapies, skin disorder therapies, small molecules, urologics, vascular disorder therapies

Calcineurin inhibitor

Oral

Inhibits calcineurin, thereby blocking various immune responses and increaseing podocyte integrity in the kidney

$\mathrm{T}_{\max } 1-4 \mathrm{~h}$, high plasma protein binding (97\%), excreted mostly in faeces, $\mathrm{t}_{1 / 2} \approx 30 \mathrm{~h}$

Decreases in eGFR, hypertension, diarrhoea, headache, anaemia, cough and urinary tract infections

L04A-D03 (voclosporin)

L4 (imunosuppressants)

(3S,6S,9S,12R,15S,18S,21S,24S,30S,33S)-30-ethyl-33-[(1R,2R,4E)-1-hydroxy-2-methylhepta-4,6dienyl]-1,4,7,10,12,15,19,25,28-nonamethyl-6,9,18,24-tetrakis(2-methylpropyl)-3,21-di(propan-2-yl)$1,4,7,10,13,16,19,22,25,28,31$-undecazacyclotritriacontane-2,5,8,11,14,17,20,23,26,29,32-undecone 
complete renal response rate at week 48 (key secondary endpoint) was significantly ( $p \leq 0.026)$ greater with lowand high-doses of voclosporin than with placebo (49.4\% and $39.8 \%$ vs $23.9 \%$ ), with OR for low-dose and high-dose voclosporin being 3.21 (95\% CI 1.68-6.13) and 2.10 (95\% CI 1.09-4.02), respectively. Although statistical significance was not reached in all subgroup analyses, a favourable complete renal response with both doses of voclosporin over placebo at weeks 24 and 48 was consistently seen regardless of sex, age, ethnicity and MMF use at screening [20].

An open-label, phase II AURION exploratory study (NCT02949973) was conducted in 10 patients with active lupus nephritis to assess the ability of biomarkers at week 8 to predict clinical response of voclosporin $23.7 \mathrm{mg}$ twice daily in combination with standard-of-care therapy at weeks 24 and 48 . Of the 10 patients who achieved $\geq 25 \%$ reduction in UPCR from baseline at week $8,80 \%$ of patients were responders (i.e. 50\% reduction in UPCR from baseline) and $70 \%$ achieved complete remission (i.e. UPCR of $0.5 \mathrm{mg} / \mathrm{mg}$, eGFR within $20 \%$ of baseline and concomitant steroid dosage of $<5 \mathrm{mg} /$ day) at week 24 [21, 22], with $71 \%$ of patients remaining in complete remission at week 48 [23].

\subsection{Adverse Events}

Voclosporin was generally well tolerated when administered as an add-on therapy to standard-of-care therapy, MMF and low dose steroids in patients with lupus nephritis in an integrated analysis of AURORA 1 and AURA-LV. The most common ( $\geq 10 \%)$ adverse events with voclosporin $23.7 \mathrm{mg}$ twice daily $(n=267)$, occurring at $\geq 2 \%$ higher than placebo $(n=266)$ included decreases in GFR (26\% vs 9\%), hypertension (19\% vs 9\%), diarrhoea (19\% vs $13 \%)$, headache (15\% vs $8 \%$ ), anaemia ( $12 \%$ vs $6 \%$ ), cough $(11 \%$ vs $2 \%)$ and urinary tract infection $(10 \%$ vs
6\%) [4]. In patients treated with voclosporin $23.7 \mathrm{mg}$ twice daily, decreases in GFR mostly occurred within the first 3 months of treatment, which mostly resolved or improved following dose modification [4]. Decreases in GFR led to permanent discontinuation of voclosporin in ten patients and was subsequently resolved in 4 of 10 patients 3 months after treatment discontinuation [4]. Overall, the incidence of serious adverse events in the AURORA 1 and AURA-LV trials was generally similar between the treatment groups $(20.8 \%$ and $28.1 \%$ with voclosporin 23.7 $\mathrm{mg}$ vs $21.3 \%$ and $15.9 \%$ with placebo), with the most common being infections [18-20]. A total of 19 patients (6 in AURORA 1 and 13 in AURA-LV) died during the study [18-20].

In voclosporin $23.7 \mathrm{mg}$ and placebo recipients, the exposure-adjusted incidence rates per 100 patient years (EAIRs/100 PYS) for serious infections were 11.9 and 12.0 , serious renal reactions were 5.6 and 3.7 , serious hypertension were 2.1 and 0.4 and serious nervous system disorders were 3.9 and 0.9 [4].

\subsection{Ongoing Clinical Trials}

A 104-week, randomized, double-blind, placebo-controlled phase III extension study AURORA 2 (NCT03597464), which is assessing the long-term efficacy and safety of voclosporin as an addition to the standard-of-care therapy in lupus nephritis in patients who had previously completed AURORA 1, has completed recruitment. The study has an estimated completion date of August 2021.

Recruitment is underway for a randomized, open-label phase II VOCOVID trial (NCT04701528), which will compare the antiviral efficacy of voclosporin versus tacrolimus in kidney transplant recipients with positive COVID-19.

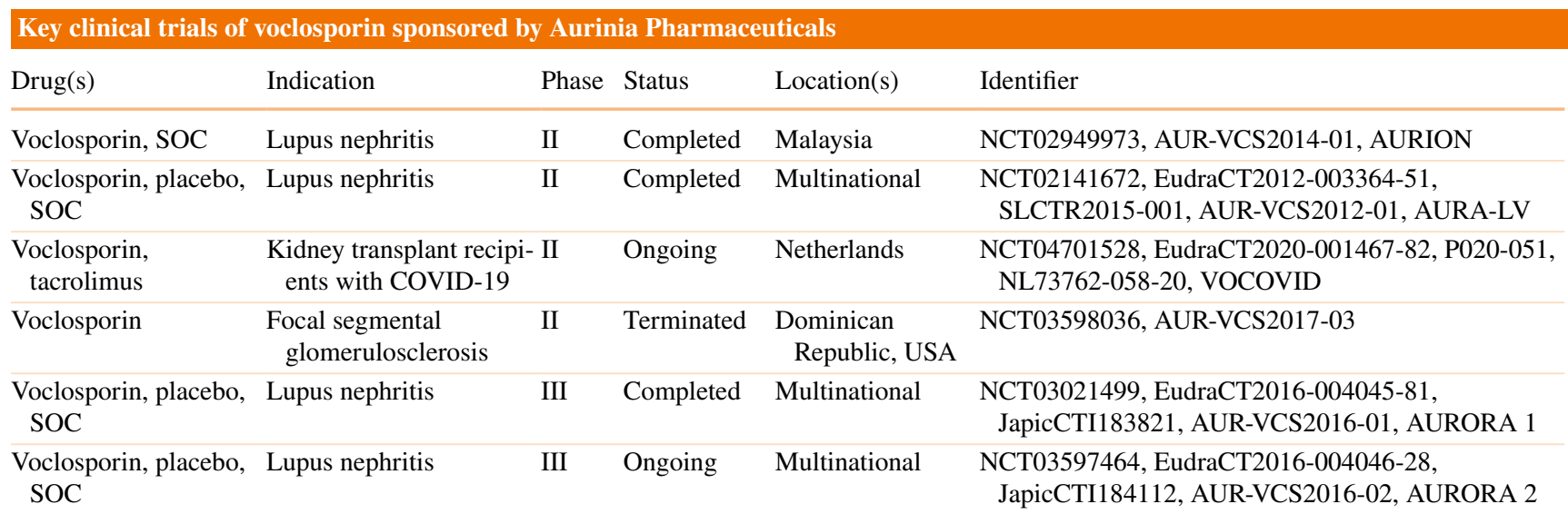

SOC standard-of-care 


\section{Current Status}

Voclosporin received its first approval on 22 January 2021 in the USA for use in combination with a background immunosuppressive therapy regimen for adults with active lupus nephritis [3].

\section{Declarations}

Funding The preparation of this review was not supported by any external funding.

Authorship and Conflict of interest During the peer review process the manufacturer of the agent under review was offered an opportunity to comment on the article. Changes resulting from any comments received were made by the authors on the basis of scientific completeness and accuracy. Young-A Heo is a salaried employee of Adis International Ltd/Springer Nature, and declares no relevant conflicts of interest. All authors contributed to the review and are responsible for the article content.

Ethics Approval, Consent to Participate, Consent to Publish, Availability of Data and Material, Code Availability Not applicable.

\section{References}

1. Fanouriakis A, Kostopoulou M, Cheema K, et al. 2019 Update of the joint European League against Rheumatism and European Renal Association-European Dialysis and Transplant Association (EULAR/ERA-EDTA) recommendations for the management of lupus nephritis. Ann Rheum Dis. 2020;79(6):S713-23.

2. Tunnicliffe DJ, Palmer SC, Henderson L, et al. Immunosuppressive treatment for proliferative lupus nephritis. Cochrane Database Syst Rev. 2018;2018(6):1-276.

3. Businesswire. FDA approves Aurinia Pharmaceuticals' Lupkynis $^{\mathrm{TM}}$ (voclosporin) for adult patients with active lupus nephritis [media release]. 25 Jan 2021. www.businesswire.com.

4. Aurinia Pharmaceuticals Inc. Lupkynis ${ }^{\mathrm{TM}}$ (voclosporin) capsules, for oral use. 2021. https://www.accessdata.fda.gov/drugsatfda docs/label/2021/213716s000lbl.pdf. Accessed 23 Feb 2021.

5. Isotechnika Pharma Inc., Aurinia Pharmaceuticals Inc. Isotechnika merger with Aurinia to create leading nephrology company [media release]. 5 Feb 2013. http://www.auriniapharma.com.

6. Aurinia Pharmaceuticals. Management's discussion and analysis of financial condtion and results of operations for the year ended December 31, 2014. 2014. https://ir.auriniapharma.com/ all-sec-filings/content/0001193125-15-112108/d891272dex993. htm. Accessed 4 Feb 2021.

7. Aurinia Pharmaceuticals. Formation of a licensing agreement covering Japan and Europe for the lupus nephritis drug "voclosporin" [media release]. 17 Dec 2020. http://www.auriniapha rma.com.

8. Aurinia Pharmaceuticals. Aurinia and Lonza announce exclusive agreement for dedicated voclosporin manufacturing capacity [media release]. 15 Dec 2020. http://www.auriniapharma. com.

9. Aurinia Pharmaceuticals. Aurinia reports third quarter 2017 financial results and provides operational highlights [media release]. 14 Nov 2017. http://www.auriniapharma.com.
10. Aurinia Pharmaceuticals. Aurinia receives notice of allowance from the US patent and trademark dffice for claims directed to its novel voclosporin dosing protocol for lupus nephritis [media release]. 25 Feb 2019. http://www.auriniapharma.com.

11. Li Y, Palmisano M, Sun D, et al. Pharmacokinetic disposition difference between cyclosporine and voclosporin drives their distinct efficacy and safety profiles in clinical studies. Clin Pharmacol. 2020;12:83-96.

12. Mayo PR, Huizinga RB, Ling SY, et al. Voclosporin food effect and single oral ascending dose pharmacokinetic and pharmacodynamic studies in healthy human subjects. J Clin Pharmacol. 2013;53(8):819-26.

13. Van Gelder T, Huizinga RB, Noukens J, et al. Use of therapeutic drug monitoring does not add clinical value for voclosporin in patients with lupus nephritis [abstract no. PO1918]. J Am Soc Nephrol. 2020;31:594.

14. Ling SY, Huizinga RB, Mayo PR, et al. Pharmacokinetics of voclosporin in renal impairment and hepatic impairment. J Clin Pharmacol. 2013;53(12):1303-12.

15. Ling SY, Huizinga RB, Mayo PR, et al. Cytochrome P450 3A and $\mathrm{P}$-glycoprotein drug-drug interactions with voclosporin. $\mathrm{Br}$ J Clin Pharmacol. 2014;77(6):1039-50.

16. van Gelder T, Huizinga RB, Lisk L, et al. Voclosporin: a novel calcineurin inhibitor without impact on mycophenolic acid in patients with SLE. Nephrol Dial Transpl. 2021. https://doi.org/ 10.1093/ndt/gfab022.

17. Caster DJ, Solomons N, Randhawa S, et al. AURORA phase 3 trial demonstrates voclosporin statistical superiority over standard of care in lupus nephritis (LN) [abstract no. MO019]. Nephrol Dial Transpl. 2020;35(Suppl 3):iii119.

18. Onno Teng YK, Parikh SV, Saxena A, et al. AURORA phase 3 study demonstrates voclosporin statistical superiority over standard of care in lupus nephritis (LN) [abstract no. 011]. Lupus Sci Med. 2020;7(Suppl 1):A14.

19. Gibson K, Parikh S, Saxena A, et al. AURORA phase 3 trial demonstrates voclosporin statistical superiority over standard of care in lupus nephritis (LN) [abstract no. 2]. Am J Kidney Dis. 2020;75(5):819.

20. Rovin BH, Solomons N, Pendergraft WF 3rd, et al. A randomized, controlled double-blind study comparing the efficacy and safety of dose-ranging voclosporin with placebo in achieving remission in patients with active lupus nephritis. Kidney Int. 2019;95(1):219-31.

21. Huizinga RB, Yahya R, Gafor AHA, et al. AURION study: 24-week data of multi-target therapy with voclosporin, MMF and steroids for active lupus nephritis [abstract no. 20]. Lupus Sci Med. 2017;4 (Suppl 1):A10-11.

22. Aurinia Pharmaceuticals. Aurinia releases open-label AURION data demonstrating increased remission rates over time for voclosporin in the treatment of lupus nephritis [media release]. 6 Oct 2016. http://www.auriniapharma.com.

23. Aurinia Pharmaceuticals. Aurinia highlights 48 -week data from open-Label AURION study at 12th international congress on SLE (LUPUS 2017) \& the 7th Asian congress on autoimmunity (ACA 2017) [media release]. 27 Mar 2017. https://ir.auriniapharma.com/ press-releases/detail/81. 